\title{
High Frequency High-Order Rayleigh Modes in $\mathrm{ZnO} / \mathrm{GaAs}$
}

\author{
J. Pedrós \\ Cavendish Laboratory, University of Cambridge \\ CB3 0HE Cambridge, United Kingdom \\ E-mail:.jp495@cam.ac.uk
}

\author{
L. García-Gancedo \\ Department of Engineering, University of Cambridge \\ CB3 0FA Cambridge, United Kingdom
}

\author{
C. J. B. Ford, C. H. W. Barnes, J. P. Griffiths, G. A. C. \\ Jones \\ Cavendish Laboratory, University of Cambridge \\ CB3 0HE Cambridge, United Kingdom
}

\author{
A. J. Flewitt \\ Department of Engineering, University of Cambridge \\ CB3 0FA Cambridge, United Kingdom
}

\author{
F. Calle \\ Instituto de Sistemas Optoelectrónicos y Microtecnología, Universidad Politécnica de Madrid, 28040 Madrid, Spain
}

\begin{abstract}
Strong high-order Rayleigh or Sezawa modes, in addition to the fundamental Rayleigh mode, have been observed in $\mathrm{ZnO} / \mathrm{GaAs}(001)$ systems along the [110] propagation direction of GaAs. The dispersion of the different acoustic waves has been calculated and compared to the experimental data. The bandwidth and impedance matching characteristics of the multimode SAW delay lines operating at high frequencies (2.53.5 $\mathrm{GHz}$ regime) have been investigated.
\end{abstract}

\section{INTRODUCTION}

$\mathrm{ZnO}$ films are known to enhance the piezoelectric coupling of GaAs substrates, facilitating the integration of surface acoustic wave (SAW) devices with the GaAs electronics. In spite of the small sound velocity mismatch between the two materials, this slow-on-fast structure supports several guided waves in the overlayer. However, only the fundamental Rayleigh wave has been studied so far $[1,2]$.

The dynamic modulation of the band diagram induced by the piezoelectric field of a SAW in GaAs-based systems has allowed the development of single-electron transistors using the split-gate technique [3]. More recently, these constrictions have been combined with lateral $n-p$ junctions pursuing a high frequency single-photon source [4]. Other approaches to SAW-assisted anti-bunched photon emitters rely on the controlled injection of excitons into quantum dots [5] or the modulation of their emission by means of the SAW strain fields [6]. All these applications would benefit strongly not only from the enhanced piezoelectric coupling provided by the $\mathrm{ZnO}$ but also from the capacity of modulating the optoelectronic systems using waves with different

This work has been partially supported by the EPSRC, grant number EP/F063865/1. J. Pedrós aknowledges the support from the Marie Curie Intra-European Fellowship within the 7th European Community Framework Programme. The ICTS Programme from the Spanish Ministerio de Ciencia e Innovación is also acknowledged. piezoelectric and strain field depth profiles to those of the fundamental Rayleigh mode.

In this paper we report on the characteristics and dispersion of the different acoustic waves supported by the $\mathrm{ZnO} / \mathrm{GaAs}$ heterostructure and on the performance of the SAW delay lines used to generate them.

\section{ZNO FILMS AND DEVICE DESIGN}

$\mathrm{ZnO}$ films of different thickness $H$, ranging from 1 to 2.5 $\mu \mathrm{m}$, were deposited on semi-insulating $\operatorname{GaAs}(001)$ substrates by high target utilization sputtering (HiTUS) [7] at room temperature. In this technique, an argon plasma is generated in a side chamber and steered onto the target, avoiding the direct contact of the plasma with the substrate. This prevents the ion bombardment of the substrate, providing high quality films with very low stress and defect density even at high deposition rates and at room temperature [7].

The sputtering parameters were optimized in order to obtain highly resistive films oriented along the (001) crystal axis, conditions that ensure a strong piezoelectricity. A Zn target $(99.999 \%)$ was exposed to the Ar plasma with a constant target power of $800 \mathrm{~W}$. The flows of Ar and $\mathrm{O}_{2}$ were 65 and $41 \mathrm{sccm}$, respectively, providing a deposition rate of $\sim 50 \mathrm{~nm} / \mathrm{min}$. The crystal quality of the sputtered $\mathrm{ZnO}$ films was assessed by X-ray diffractometry, confirming the unique orientation of the films along the (001) axis. The resistivity of all the films was very high $\left(10^{10} \Omega \mathrm{m}\right)$.

SAW delay lines, formed by split-finger interdigital transducers (IDTs), were patterned by e-beam lithography on 
the $\mathrm{ZnO}$ films along the [110] direction of the underlying $\mathrm{GaAs}(001)$ substrates. The IDTs were formed by $\mathrm{Ti} / \mathrm{Au}(10 / 10$ $\mathrm{nm})$ contacts and had a period $\lambda$ of $1 \mu \mathrm{m}$ and a metallization ratio of 0.5 , i.e. finger width and pitch of $125 \mathrm{~nm}$. The IDTs had an aperture $W$ of $60 \mu \mathrm{m}$, whereas the number of finger pairs $N_{p}$ was varied in order to modify the bandwidth of the passband. The delay lengths were 1 and $2 \mathrm{~mm}$. The $\mathrm{S}$ parameters of the devices were measured with a coplanar probe station connected to a network analyzer, and in some cases (when specifically stated) the devices were also characterized once mounted on a sample holder with SMA connectors.

\section{RESULTS}

\section{A. High-order Rayleigh or Sezawa modes}

Fig. 1 shows the insertion loss of identical SAW delay lines fabricated on a series of $\mathrm{ZnO}$ films of different thickness on $\mathrm{GaAs}(001)$ substrates. The spectra of the devices on films with thickness $H$ ranging from 1 to $1.4 \mu \mathrm{m}$ present two clear resonances, whereas the device on the thickest film $(H=2.5 \mu \mathrm{m})$ supports three. The lowest-frequency resonance, at $2.53 \mathrm{GHz}$ for all the devices, corresponds to the fundamental Rayleigh mode (labeled as R). The resonances at higher frequencies are associated with high-order Rayleigh modes, also called Sezawa modes (labeled as $S_{i}, i \geq 1$ ). These are guided waves in the overlayer that arise since the $\mathrm{ZnO} / \mathrm{GaAs}$ structure forms a slow-on-fast system, i.e. the bulk transverse velocity in the substrate is larger than in the overlayer. The frequency of the first Sezawa mode $\left(\mathrm{S}_{1}\right)$ decreases from 3.20 to $2.86 \mathrm{GHz}$ as the film thickness increases from 1 to $2.5 \mu \mathrm{m}$ (as indicated by the arrow in Fig.1), whereas the second Sezawa mode $\left(\mathrm{S}_{2}\right)$ appears only for the thickest film, at a frequency of $3.08 \mathrm{GHz}$.

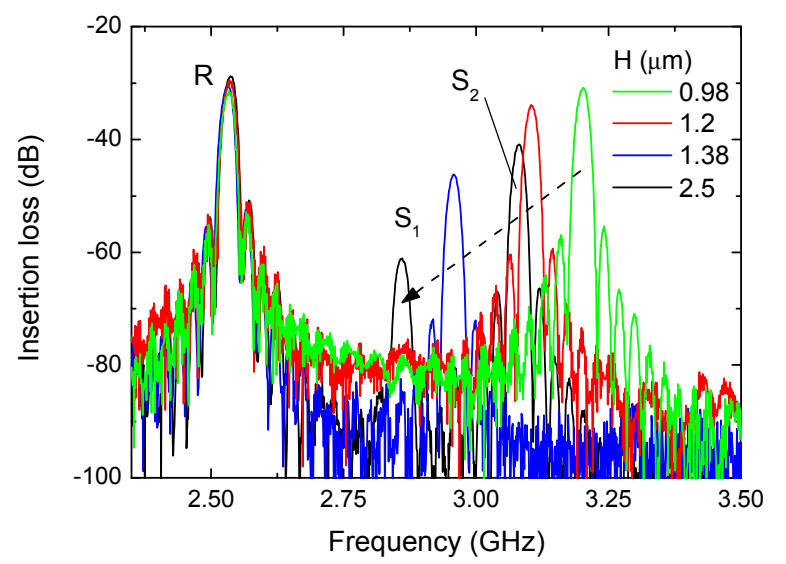

Figure 1. Insertion loss of identical SAW delay lines on $\mathrm{ZnO}$ films of different thickness $H$ on GaAs. R, $\mathrm{S}_{1}$, and $\mathrm{S}_{2}$ denote the Rayleigh and the first and second Sezawa modes, respectively.

\section{B. Dispersion of the $\mathrm{ZnO} / \mathrm{GaAs}$ structure}

The identification of the acoustic modes shown in Fig. 1 is based on the comparison of the experimental data with the numerical calculation of the dispersion of the $\mathrm{ZnO} / \mathrm{GaAs}$ structure. The calculation is based on a Green's function formalism which was developed to calculate Brillouin spectra [8]. The material parameters of $\mathrm{ZnO}$ and $\mathrm{GaAs}$ used for the calculation have been taken from Refs. [9] and [10], respectively.

The results of the simulations for the $\mathrm{ZnO} / \mathrm{GaAs}(001)$ heterostructure with propagation along the GaAs [110] direction are summarized in Fig. 2. $\mathrm{ZnO}$ has a wurzite structure (crystal class $6 \mathrm{~mm}$ ) with isotropic elastic properties in the $(00.1)$ plane, so that the propagation direction is referred to that of the underlying cubic structure of GaAs (crystal class $-43 \mathrm{~m}$ ) that presents a four-fold symmetry in the elastic properties in the (001) plane. The gray scale in Fig. 2 depicts the magnitude of the shear vertical component of a wave having the given velocity and $k H=2 \pi H / \lambda$ value. The experimental data (circles) have been determined from the resonance frequency of the IDTs (Fig. 1). The velocity of the $\mathrm{R}$ mode decreases from its value in $\mathrm{GaAs}$ for $k H=0$, to that in $\mathrm{ZnO}$ for approximately $k H>7$. Additionally, $\mathrm{S}_{\mathrm{i}}$ modes emerge nearly periodically beyond their threshold values of $k H$. The velocity of the $\mathrm{S}_{\mathrm{i}}$ modes decreases monotonically with increasing $k H$. The numerical results are in good agreement with the experimental data. The small discrepancy on the $\mathrm{S}_{\mathrm{i}}$ modes in the thickest $\mathrm{ZnO}$ film could originate from a slight misalignment of the delay line pattern from the [110] direction. The broadening of the $\mathrm{R}$ mode is an artifact of the calculation when resolving simultaneously the intense $\mathrm{R}$ mode and the less intense $\mathrm{S}_{\mathrm{i}}$ modes.

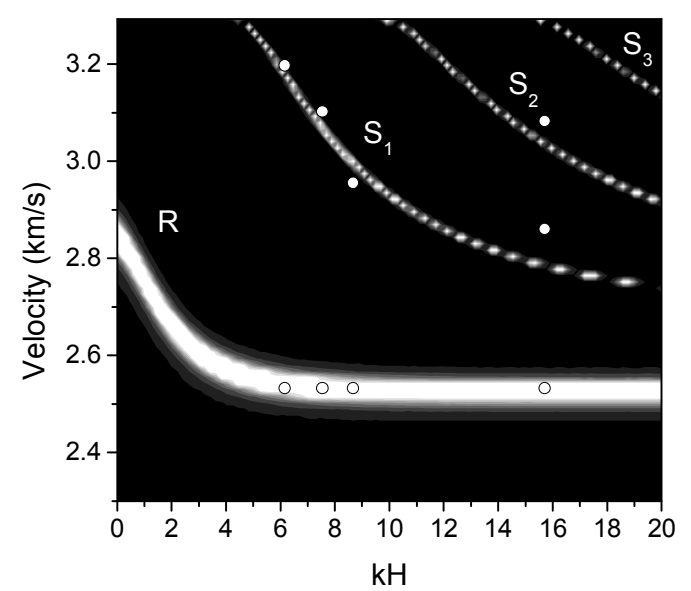

Figure 2. Dispersion of the Rayleigh $(\mathrm{R})$ and Sezawa $\left(\mathrm{S}_{\mathrm{i}}\right)$ modes in the $\mathrm{ZnO} / \mathrm{GaAs}(001)$ structure for propagation along the [110] direction of GaAs. The brighter the scale is, the larger the calculated intensity of the mode. The experimental data are shown by the circles.

\section{Device bandwidth}

Figs. 3(a) and 3(b) depict, respectively, the insertion and return loss of a series of SAW delay lines with varying number of finger pairs $\left(N_{p}\right)$ fabricated on a $2.5 \mu \mathrm{m}$-thick $\mathrm{ZnO}$ film on $\operatorname{GaAs}(001)$. The resonances of the $\mathrm{R}, \mathrm{S}_{1}$, and $\mathrm{S}_{2}$ modes are clearly observed in both the transmission and the reflection spectra for all the cases except for the $S_{1}$ mode in the reflection spectra for the lower $N_{p}$ cases, where it is hardly distinguished from the background. 

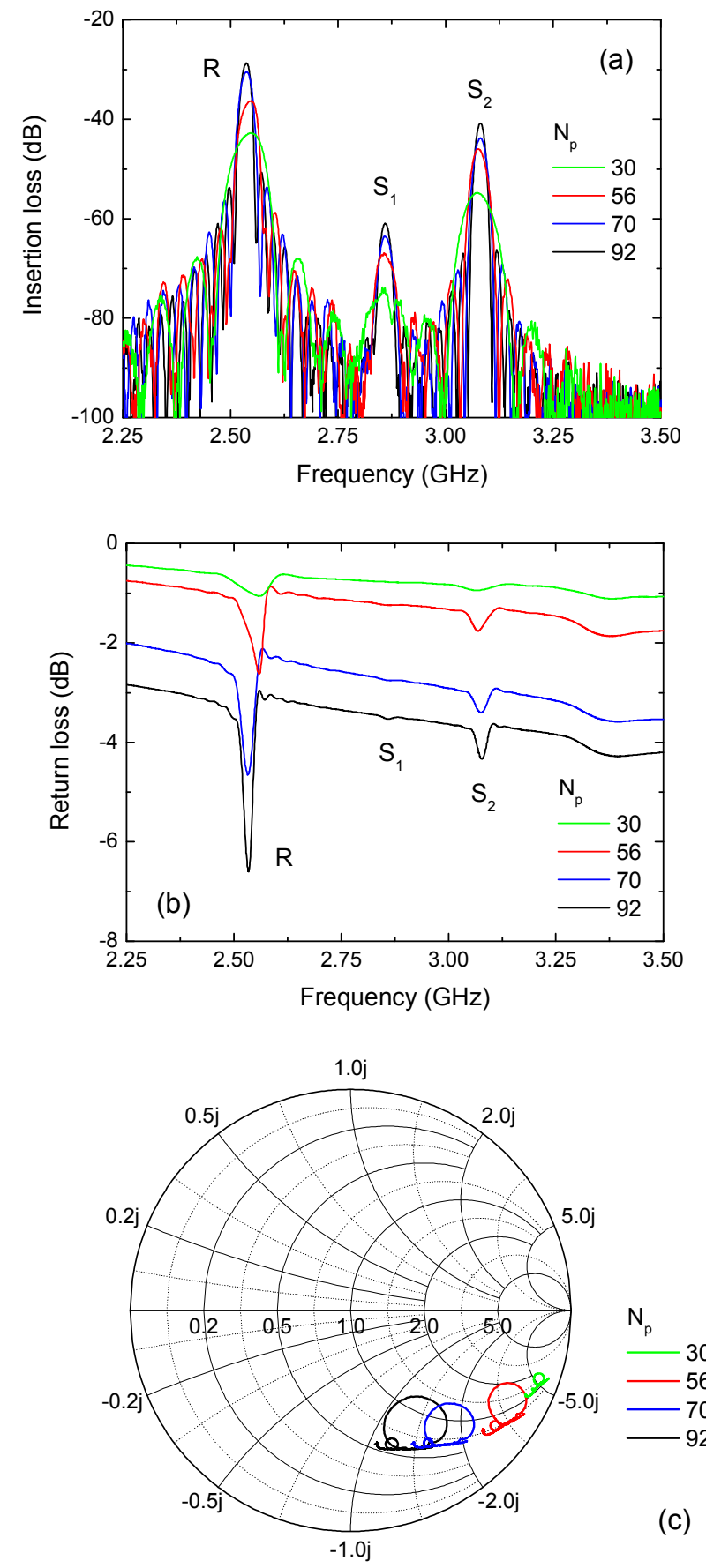

Figure 3. (a) Insertion loss, (b) return loss, and (c) Smith chart (normalized to $50 \Omega$ ) of SAW delay lines with varying number of finger pairs $N_{p}$ on a 2.5$\mu \mathrm{m}$ thick $\mathrm{ZnO}$ film on GaAs. $\mathrm{R}, \mathrm{S}_{1}$, and $\mathrm{S}_{2}$ denote the Rayleigh and the first and second Sezawa modes, respectively.

The variation of $N_{p}$ modifies the losses of the devices by means of two different mechanisms. On the one hand, the passband of the devices narrows as $N_{p}$ increases and this translates into a reduction of the losses. This correlation is clearly observed in Figs. 3(a) and 3(b). On the other hand, the increase in $N_{p}$ produces an increase in the capacitance of the IDT, decreasing its reactance and thus enhancing the impedance matching condition of the device. Fig. 3(c) shows the Smith chart of the impedance of the devices. The traces, in the capacitive half of the chart, move approximately along curves of constant resistance, except at the mode resonances, where a loop is formed. As $N_{p}$ increases, the traces present larger loops, i.e. stronger resonances, and they shift towards smaller values of capacitive reactance.

The evolution of the bandwith with $N_{p}$ for the different mode resonances shown in Fig. 3(a) is summarized in Fig. 4. Both, the bandwidth between first nulls on either side of the resonance frequency $\left(B W_{n n}\right)$ and between points $3 \mathrm{~dB}$ below the main lobe maximum $\left(B W_{3}\right)$ have been extracted from the experimental data in Fig. 3(a). The experimental bandwidths in Fig. 4 (labeled as $B W_{n n}$ exp and $B W_{3 \text { exp }}$ ) are compared to their theoretically expected values (labeled as $B W_{n n}$ th and $B W_{3}$ th). The theoretical bandwidth between first nulls is given by the expression $B W_{n n}=2 f_{0} / N_{p}$, where $f_{0}$ is the resonance frequency of the mode. The theoretical value of the bandwidth at $3 \mathrm{~dB}$ is $B W_{3} \approx 0.31 B W_{n n}$, the characteristic value of a square sinc function at that level. The comparison of the experimental and theoretical values of $B W_{3}$ shows an overall good agreement, whereas the experimental values of $B W_{n n}$ are systematically lower than the theoretically predicted ones. This discrepancy is likely to be related to the error in determining the position of the nulls. This error is especially large in the case of the $\mathrm{S}_{1}$ mode where first nulls appear at a very high insertion loss, as can be noticed in Fig. 3(a).

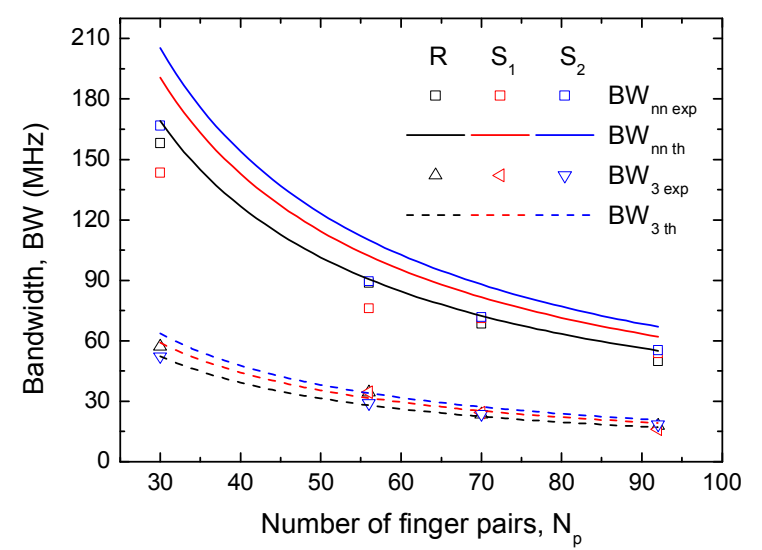

Figure 4. Evolution of the bandwidth of the Rayleigh (R), first Sezawa $\left(S_{1}\right)$, and second Sezawa $\left(S_{2}\right)$ mode resonances with the number of finger pairs in SAW delay lines on a $2.5-\mu \mathrm{m}$ thick $\mathrm{ZnO}$ film on GaAs. See text for details.

\section{Impedance matching}

A stub tuner has been used to impedance match one of the IDTs forming the SAW delay lines under study in order to determine the maximum potential of the devices. The selected device was patterned on a $2.5 \mu \mathrm{m}$-thick $\mathrm{ZnO}$ film on $\operatorname{GaAs}(001)$ formed by IDTs with $W=60 \mu \mathrm{m}, \lambda=1 \mu \mathrm{m}$, and $N_{p}=70$. The device was mounted on a sample holder with SMA connectors. The contribution of the measurement set-up, including an SMA cable and the stub tuner in its initial contracted position, has been eliminated by means of the calibration. 
Fig. 5 shows the return loss spectra of an IDT before and after using the stub tuner to impedance match it, whereas the inset presents the data in the format of a Smith chart, where the variation of the impedance with frequency is depicted. The comparison of the spectra in Fig. 5 and its inset for the untunned IDT with those of a similar IDT $\left(N_{p}=70\right)$ in Figs. 3(b) and 3(c) indicates the contribution of the sample holder. The traces, which are in the capacitive half of the chart when measured on wafer with probes [see Fig. 3(c)], cross over from inductive to capacitive when measured on housing, as shown in the inset of Fig. 5. In particular, the resonance of the $\mathrm{R}$ mode lies in the inductive half of the chart. The inductance measured is likely to originate from the wire bondings used to connect the device to the sample holder.

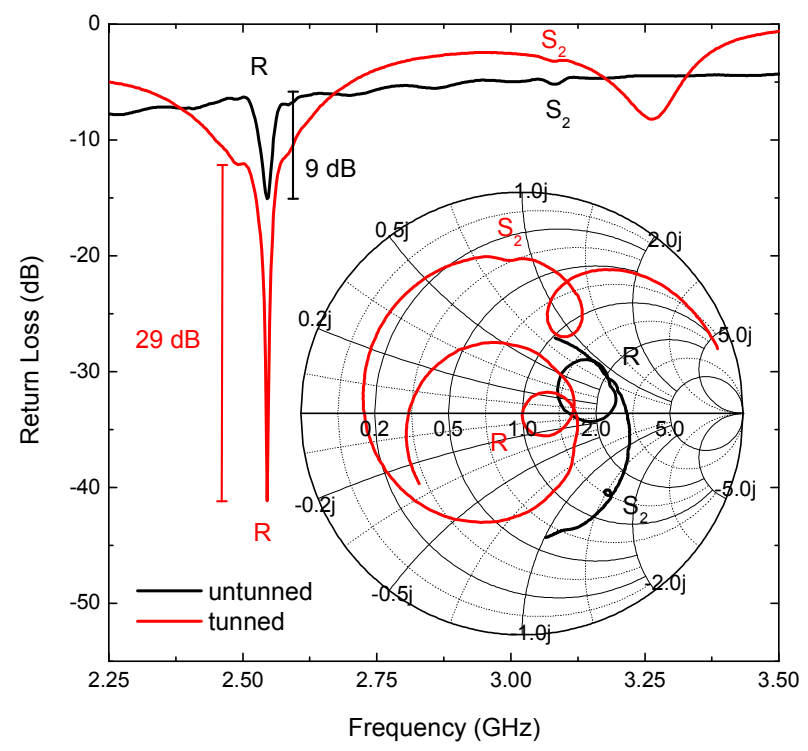

Figure 5. Return loss of an IDT with $N_{p}=70$ on a $2.5-\mu \mathrm{m}$ thick $\mathrm{ZnO}$ film on GaAs (device mounted on a sample holder). The inset shows the Smith chart (normalized to $50 \Omega$ ) of the return loss. $\mathrm{R}$ and $\mathrm{S}_{2}$ denote the Rayleigh and second Sezawa modes, respectively.

When the stub tuner is used to impedance match the $\mathrm{R}$ mode, as indicated by its resonance centered at 1 on the real axis (i.e. $50 \Omega$ ) of the Smith chart (inset of Fig. 5), the deep associated to that mode in the return loss spectrum (Fig. 5) is enhanced by more than $25 \mathrm{~dB}$. Nonetheless, the stub tuner introduces an additional modulation of the background of approximately $8-10 \mathrm{~dB}$ in amplitude and a period of $715 \mathrm{MHz}$. One of the minima of this modulation coincides with the resonance of the $\mathrm{R}$ mode, broadening its base. If that contribution is subtracted, the net enhancement of the magnitude of the $\mathrm{R}$ mode amounts to $20 \mathrm{~dB}$, as indicated in Fig. 5.

\section{CONCLUSIONS}

Strongly oriented and highly resistive $\mathrm{ZnO}$ films on GaAs substrates have been obtained by sputtering at room temperature. The different acoustic waves propagating along the [110] direction of $\mathrm{GaAs}$ in $\mathrm{ZnO} / \mathrm{GaAs}(001)$ systems have been experimentally and theoretically investigated. In addition to the fundamental Rayleigh mode, strong high-order Rayleigh or Sezawa modes propagate confined in the overlayer. In particular, the insertion loss of the $S_{1}$ mode can be as low as that of the $\mathrm{R}$ mode for certain thickness-to-wavelength ratios. The bandwidth and impedance matching characteristics of the different resonances in SAW delay lines operating at high frequency (2.5-3.5 GHz regime) have been investigated.

\section{REFERENCES}

[1] Y. Kim, W. D. Hunt, F. S. Hickernell, R. J. Higgins, and C.-K. Jen, "ZnO films on $\{001\}$-cut $<110>$-propagating GaAs substrates for surface acoustic wave device applications" IEEE Trans. Ultrason. Ferroelec. Cont., vol. 42, pp. 351- 361, 1995.

[2] V. Y. Zhang, J. E. Lefebvre, and T. Gryba, "SAW characteristics in a layered $\mathrm{ZnO} / \mathrm{GaAs}$ structure for design of integrated SAW filters" Proc. IEEE Ultrason. Symp., pp. 261-264, 2001.

[3] J. M. Shilton, V. I. Talyanskii, M. Pepper, J. E. F. Frost, C. J. B. Ford, C. G. Smith, and G. A. C. Jones, "High-frequency single-electron transport in quasi-one-dimensional GaAs channel induced by surface acoustic waves", J. Phys. Condens. Matter, vol. 8, pp. L531-L539, 1996.

[4] J. R. Gell, P. Atkinson, S. P. Bremner, F. Sfigakis, M. Kataoka, D. Anderson, G. A. C. Jones, C. H. W. Barnes, D. A. Ritchie, M. B. Ward, C. E. Norman, and A. J. Shields, "Surface-acoustic-wave-driven luminescence from lateral $p-n$ junction", Appl. Phys. Lett., vol. 89, pp. 243505-1-4, 2006.

[5] O. D. D. Couto Jr., S. Lazic, F. Iikawa, J. A. H. Stotz, U. Jahn, R. Hey, and P. V. Santos, "Photon anti-bunching in acoustically pumped quantum dot" Nat. Photonics, vol. 3, pp. 645-648, 2009.

[6] J. R. Gell, M. B. Ward, R. J. Young, R. M. Stevenson, P. Atkinson, D. Anderson, G. A. C. Jones, D. A. Ritchie, and A. J. Shields, "Modulation of single quantum dot energy levels by a surface-acousticwave”, Appl. Phys. Lett., vol. 93, pp. 018115-1-4, 2008.

[7] L. García-Gancedo, J. Pedrós, A. J. Flewitt, W. I. Milne, G. M. Ashley, J. Luo, and C. J. B. Ford, "Ultrafast sputtered $\mathrm{ZnO}$ thin films with high $k_{T}$ for acoustic wave device applications" Proc. IEEE Ultrasonics Symp. 2010, in press.

[8] X. Zhang, J. D. Comins, A. G. Every, P. R. Stoddart, W. Pang, and T. E. Derry, "Surface Brillouin scattering study of the suface excitations in amorphous silicon layers produced by ion bombardment", Phys. Rev. B, vol. 58, pp. 13677- 13685, 1998.

[9] G. Carlotti, G. Socino, A. Petri, and E. Verona, "Acoustic investigation of the elastic properties of $\mathrm{ZnO}$ films" Appl. Phys. Lett., vol. 51, pp. 1889-1891, 1987.

[10] A. J. Slobodnik, Microwave Acoustics Handbook, 1973. 